
cultures

Les cahiers de l'Acedle

6-2 | 2009

Didactique des langues et linguistique

\title{
L'insertion du CECR en Albanie
}

\section{Eldina Nasufi}

\section{OpenEdition}

\section{Journals}

Édition électronique

URL : http://journals.openedition.org/rdlc/1925

DOI : $10.4000 /$ rdlc. 1925

ISSN : 1958-5772

Éditeur

ACEDLE

\section{Référence électronique}

Eldina Nasufi, "L'insertion du CECR en Albanie », Recherches en didactique des langues et des cultures [En ligne], 6-2 | 2009, mis en ligne le 01 octobre 2009, consulté le 19 avril 2019. URL : http:// journals.openedition.org/rdlc/1925; DOI : 10.4000/rdlc.1925

Ce document a été généré automatiquement le 19 avril 2019

\section{(†)

Recherches en didactique des langues et des cultures is licensed under a Creative Commons AttributionNonCommercial-NoDerivatives 4.0 International License 


\title{
L'insertion du CECR en Albanie
}

\author{
Eldina Nasufi
}

\section{La politique linguistique et la situation actuelle en Albanie}

1 La politique linguistique menée en Albanie se donne pour objectifs l'enseignement de la langue albanaise (langue nationale de l'Albanie), des langues étrangères et des langues des minorités. Avant les années 90 , les langues étrangères étaient considérées comme une fenêtre d'où l'on pouvait voir une toute petite partie du monde, mais à l'école on n'a jamais appris que l'anglais, le français et le russe (jusqu'à la fin des années 80 ). De nos jours on apprend l'anglais, le français et l'italien. Les événements politiques des années 90, l'ouverture de l'Albanie ont renforcé l'importance de l'enseignement / apprentissage des langues. Les Albanais, à partir de cette période ont été libres de travailler et de vivre à l'étranger. Un grand nombre de jeunes choisissent de faire leurs études dans les pays européens ou dans d'autres pays encore.

2 On élabore de plus en plus de nouveaux curricula, on s'occupe particulièrement de la formation des professeurs car on retient l'enseignement comme axe prioritaire. Les anciennes méthodes, les anciens manuels avec lesquels on apprenait les langues, avec des méthodes de grammaire et traduction en particulier, tendent à être remplacés par des méthodes communicatives. Les langues des minorités sont enseignées de l'école primaire jusqu'à l'université notamment la langue grecque.

3 L'Europe représente encore un défi majeur pour les Albanais et des efforts sérieux sont réalisés pour pouvoir s'y intégrer. De plus en plus d'organismes internationaux sont présents dans le pays.

4 Deux événements ont marqué un tournant important pour les politiques linguistiques en général et pour le processus d'enseignement / apprentissage des langues étrangères en Albanie, à savoir :

- l'introduction d'exigences convenant aux relations avec le Conseil de l'Europe ;

- la mise en route du processus d'association et de stabilisation. 
Les retombées de ces développements ne pouvaient être que positives pour le domaine des langues. Depuis ces événements il y a eu un très grand souci de travailler et d'élaborer des curricula fondés sur les principes du Cadre européen commun de référence (CECR, Conseil de l'Europe, 2001). D'ailleurs un très grand travail est fait au niveau universitaire pour être cohérent avec les principes de la convention de Bologne. En guise d'exemple, nous prendrons le cas de la faculté des langues étrangères à Tirana où, actuellement, les étudiants ont le droit d'apprendre trois langues étrangères. Les différents modules sont organisés selon le système des crédits. Cette année sont implémentés également les diplômes du deuxième niveau.

\section{Certains principes de base du CECR}

6 Nous allons dans ce qui suit commenter quelques-uns des principes de base du Cadre européen commun de référence. Celui-ci offre une base commune pour l'élaboration de programmes de langues vivantes, de référentiels, d'examens, de manuels, etc. en Europe. Il décrit aussi complètement que possible ce que les apprenants d'une langue doivent apprendre afin de l'utiliser dans le but de communiquer ; il énumère également les connaissances et les habiletés qu'ils doivent acquérir afin d'avoir un comportement langagier efficace. La description englobe aussi le contexte culturel qui soutient la langue. Enfin, le Cadre de référence définit les niveaux de compétence qui permettent de mesurer le progrès de l'apprenant à chaque étape de l'apprentissage et à tout moment de la vie.

7 Le Cadre européen commun de référence est conçu pour que soient surmontées les difficultés de communication rencontrées par les professionnels des langues vivantes et qui proviennent de la différence entre les systèmes éducatifs. Le Cadre donne des outils aux administrateurs, aux concepteurs de programmes, aux enseignants, à leurs formateurs, aux jurys d'examens, etc., pour réfléchir à leur pratique habituelle afin de situer et de coordonner leurs efforts et de garantir qu'ils répondent aux besoins réels des apprenants dont ils ont la charge. En fournissant une base commune à des descriptions explicites d'objectifs, de contenus et de méthodes, le Cadre de référence améliorera la transparence des cours, des programmes et des qualifications, favorisant ainsi la coopération internationale dans le domaine des langues vivantes. Donner des critères objectifs pour décrire la compétence langagière facilitera la reconnaissance mutuelle des qualifications obtenues dans des contextes d'apprentissage divers et, en conséquence, ira dans le sens de la mobilité en Europe. Le choix pour le Cadre d'une présentation taxonomique constitue, à coup sûr, une tentative pour traiter la grande complexité du langage humain en découpant la compétence langagière selon ses différentes composantes. Ceci nous renvoie à des problèmes psychologiques et pédagogiques d'importance. La communication met tout l'être humain en jeu. Les compétences isolées et classifiées dans le CECR se combinent de manière complexe pour faire de chaque individu un être unique. En tant qu'acteur social, chaque individu établit des relations avec un nombre toujours croissant de groupes sociaux qui se chevauchent et qui, tous ensemble, définissent une identité. Dans une approche interculturelle, un objectif essentiel de l'enseignement des langues est de favoriser le développement harmonieux de la personnalité de l'apprenant et de son identité en réponse à l'expérience enrichissante de l'altérité en matière de langue et de culture. Il revient aux enseignants et aux apprenants eux-mêmes de construire une personnalité saine et équilibrée à partir des éléments variés qui la composeront. Le Cadre de référence comprend la description de qualifications "partielles » 
qui conviennent à une connaissance réduite de la langue (par exemple, s'il s'agit plus de comprendre que de parler) ou lorsque le temps disponible pour l'apprentissage d'une troisième ou d'une quatrième langue est limité et que des résultats plus rentables peuvent éventuellement être atteints en visant, par exemple, la reconnaissance plutôt que des habiletés fondées sur la mémoire. La reconnaissance formelle de capacités de ce type aidera à promouvoir le plurilinguisme par l'apprentissage d'une plus grande variété de langues européennes.

Le Cadre européen commun de référence servira notamment à :

élaborer des programmes d'apprentissage des langues prenant en compte

- les savoirs antérieurs supposés acquis et l'articulation de ces programmes avec les apprentissages précédents, notamment aux interfaces entre le primaire, le premier cycle du secondaire, le second cycle du secondaire, l'enseignement supérieur et l'enseignement continu ;

- les objectifs ;

- les contenus ;

organiser une certification en langues à partir

- d'examens définis en termes de contenu ;

- de critères d'appréciation formulés en termes de résultats positifs, plutôt qu'en soulignant les insuffisances ;

mettre en place un apprentissage autodirigé qui consiste à

- développer chez l'apprenant la prise de conscience de l'état présent de ses connaissances et de ses savoir-faire ;

- l'habituer à se fixer des objectifs valables et réalistes ;

- lui apprendre à choisir du matériel ;

- l'entraîner à l'auto-évaluation.

\section{Le CECR, réel support d'étude ? Son impact dans les pratiques professionnelles des enseignants}

L'an 2000 constitue le point de départ pour la sensibilisation au CECR. Dans cet objectif ont été organisés différents types d'activités. Pendant cette période on a mis en évidence l'importance du CECR et de la rédaction des portfolios de langues en albanais. Nombre de professeurs et de chercheurs ont publié des articles à ce sujet dans les revues les plus prestigieuses du pays telles que Buletini Shkencor de Shkodër, Kërkime universitare de Gjirokastër, Revista Pedagogjike de Tirana, Buletini Shkencor d'Elbasan. Ces écrits constituent des efforts dans le cadre de l'explicitation du Cadre en tant que pratique nouvelle dans l'enseignement / apprentissage des langues étrangères.

Des formations pour de futurs formateurs ont eu lieu dans l'Institut des curricula et des standards, il s'agit de formations assurées par les spécialistes du Conseil de l'Europe. Les formations poursuivaient un double objectif : former les futurs formateurs et les sensibiliser au plurilinguisme. Nous avons eu nous-même, la possibilité de faire partie du groupe qui a été formé pour devenir formateur et pour traduire le portfolio en albanais. Ce travail s'est révélé très exigeant mais très utile pour le contexte albanais. D'autres professeurs d'autres langues à l'université ont collaboré et ont réparti le travail en groupes pour pouvoir mener au succès cette initiative ambitieuse. Actuellement le passeport et les descripteurs ont été traduits en albanais et chaque professeur peut avoir recours à ce support pour concevoir ses cours. 
11 En ce qui concerne l'Alliance Française de Tirana, qui constitue le plus grand centre où l'on apprend le français par rapport aux autres alliances de villes telles que Shkodra, Elbasan et Korça, il y a eu deux séminaires de formation au CECR et aux descripteurs. Cela fait trois ans désormais qu'à l'Alliance Française de Tirana on délivre les diplômes DELF / DALF (Diplôme d'Études en Langue Française, Diplôme Approfondi de Langue Française) calibrés en fonction des échelles du CECR. Ce contingent de professeurs a été formé également pour devenir correcteurs pour les examens DELF / DALF. Grâce à ces formations organisées par le CIEP (Centre International d'Études Pédagogiques) et la certification des diplômes, un très grand nombre d'apprenants s'inscrivent dans les cours préparatoires DELF / DALF et se soumettent à ces épreuves dans le but d'entreprendre des études en France.

12 Malgré l'important travail qui a été accompli pour faire connaître le CECR et le portfolio auprès d'un grand nombre d'enseignants, peu d'entre eux utilisent pour l'instant le CECR dans leurs cours.

13 Si nous nous référons au niveau universitaire, il y a encore des lacunes dans la référence réelle au CECR. Avec la mise en place de la convention de Bologne, on a mis l'accent sur l'importance d'une évaluation des langues étrangères selon les descripteurs du Cadre, surtout pour la deuxième et la troisième langue étrangère que les étudiants acquièrent a l'université. Mais actuellement on évalue encore les étudiants en se référant aux anciennes méthodes d'évaluation. Cette évaluation ne s'établit pas en fonction des six niveaux du cadre. Quand on délivre les diplômes universitaires, les compétences en langue étrangère ne figurent pas explicitement, il y a seulement un relevé de notes. Donc il n'y a pas encore d'évaluation prenant en compte les échelles du CECR.

Dans les programmes conçus pour le premier et le deuxième cycle d'études, dans aucun des départements de langue étrangère d'Albanie, on ne trouve de modules qui portent sur le CECR ou sur des questions importantes traitées dans le CECR telles que l'interculturel. Ce dernier aspect est traité comme une question incorporée dans des matières du type "Civilisation » ou « Anthropologie culturelle et sociale », mais pas comme une matière à part dans le cadre d'une didactique des langues et des cultures.

15 Le problème majeur reste celui des manuels de langue étrangère. Au niveau universitaire, on utilise encore des méthodes comme Bonne route (Gibert \& Greffet, 1988-1990) et Nouveau sans frontières (Girardet et al., 1988-1990) pour les étudiants dont le français est la deuxième ou la troisième langue étrangère.

Dans les lycées bilingues on utilise les méthodes Café Crème (Beacco di Giura et al., 1997-2000) ou Campus (Girardet \& Pécheur, 2002-2006). Dans les lycées à caractère général on trouve des manuels conçus par des auteurs albanais. Ces manuels ont été élaborés avant que leurs auteurs soient formés au Cadre. Il en est de même pour l'enseignement primaire, la plupart des méthodes utilisées sont celles conçues par des auteurs albanais. Ainsi un des plus grands problèmes à résoudre est le remplacement des anciens manuels de langues par des manuels qui répondent aux exigences du CECR.

17 Seule l'Alliance Française de Tirana utilise des manuels calibrés selon les échelles du cadre, tels que Alter Ego (Berthet et al., 2006-2008). Précédemment l'Alliance Française de Tirana avait utilisé pendant quatre ans la méthode Tempo (Bérard et al., 1996-2001).

Tous les professeurs ne sont pas formés au Cadre de manière à être incités à refléter celuici dans leurs pratiques de classe. Il y a encore du travail à faire sur ce point, surtout si l'on pense que l'élaboration d'un manuel de FLE (Français Langue Étrangère) ou d'une autre 
langue étrangère n'est pas une tâche facile. Il faudrait également tenir compte d'un autre acteur que sont les maisons d'édition. Il semble que, en théorie, les professeurs ont pris conscience que le CECR est devenu une référence incontournable, mais les choix de documents, d'activités et de tâches ne correspondent pas aux critères du Cadre. Ce sont les manuels existants qui déterminent les pratiques professionnelles. Les pratiques du type jeux de rôle, simulations, travail en équipe, etc. trouvent très peu de place dans les cours. Ajoutons à cela les difficultés des professeurs à trouver les conditions minimales pour pouvoir organiser les activités proposées dans le Cadre. L'utilisation du CECR reste encore disparate dans l'ensemble du pays et également dans la capitale.

\section{BIBLIOGRAPHIE}

Beacco di Giura, M., Canelas, J., Delaisne, P., Jennepin, D., Kaneman-Pougatch, M., Mcbride, N., Massacret, É., Mothe, P., Trevisi, S. \& Pons, S. (1997-2000). Café Crème. Paris : Hachette.

Berthet, A., Daill, E., Dollez, C., Hugot, C., Kizirian, V., Pons, S., Sampsonis, B. \& Waendendries, M. (2006-2008). Alter Ego. Paris : Hachette.

Bérard, É., Canier, Y. \& Lavenne, C. (1996-2001). Tempo. Paris : Didier.

Conseil de l'Europe (2001). Cadre européen commun de référence pour les langues - Apprendre, enseigner, évaluer. Paris : Didier. Disponible en ligne : http://www.coe.int/fr/web/portfolio/thecommon-european-framework-of-reference-for-languages-learning-teaching-assessment-cefr-

Gibert, P. \& Greffet, P. (1988-1990). Bonne route 1 - Méthode de français. Paris : Hachette.

Girardet, J., Dominique, P., Cridlig, J.-M., Plum De Vries, C., Vassal, J., Verdelhan, M. \&

Verdelhan, M. (1988-1990). Nouveau sans frontières. Paris : Clé international.

Girardet, J. \& Pécheur, J. (2002-2006). Campus. Paris : Clé international.

\section{RÉSUMÉS}

Dans cet article nous avons l'intention de faire l'état des lieux des démarches et des efforts qui ont été réalisés pour insérer le Cadre Commun de Référence pour les langues en Albanie. Dans cet objectif nous allons essayer de voir si vraiment le CECR s'applique dans les pratiques des enseignants albanais, quelles sont les réussites et quels sont les problèmes que les enseignants rencontrent concernant cette question si importante dans le domaine de l'enseignement / apprentissage des langues.

Nous allons nous concentrer en particulier sur l'enseignement / apprentissage du français dans tous les niveaux de l'enseignement et en même temps nous nous pencherons sur le cas de l'Alliance Française de Tirana où un très large public de différents âges apprend la langue française et se prépare pour entreprendre des études en France.

In this article, we aim to describe the attempts made to introduce the Common European Framework of Reference for Languages in Albania. With this purpose in mind, we will try to establish whether the CEFR can really be applied in the practice of Albanian teachers, and to 
identify the achievements and the problems teachers face in relation to this important issue in the field of language teaching/learning.

We will concentrate especially on the teaching/learning of French language at all levels of teaching and at the same time we will focus on the case of the Alliance Française, in Tirana where a significant number of people of different age groups learn the French language and prepare to initiate their studies in France.

\section{INDEX}

Mots-clés : CECR, formation, sensibilisation

Keywords : CEFR, training, sensibilisation

\section{AUTEUR}

\section{ELDINA NASUFI}

Eldina Nasufi enseigne le français à la faculté des langues étrangères de l'université de Tirana, en Albanie. Son domaine de spécialisation est la didactique et la grammaire textuelle. Elle a soutenu un master 2 recherche en didactique des langues et des cultures à l'université Paris III Sorbonne Nouvelle et est actuellement inscrite en thèse dans son département en Albanie.

Courriel : Eldina_n[at]yahoo.com

Adresse : Universiteti i Tiranes, Fakulteti i Gjuheve te Huaja, Rruga e Elbasanit, Tirana, Albanie. 